\title{
A Progressive Deployment Strategy for Cooperative Adaptive Cruise Control to Improve Traffic Dynamics
}

\author{
Georges M. Arnaout ${ }^{1}$ Shannon Bowling ${ }^{2}$ \\ ${ }^{1}$ Department of Engineering Management and Systems Engineering, Old Dominion University, Norfolk, VA 23529, USA \\ ${ }^{2}$ Department of Electrical Engineering Technology, Bluefield State College, Bluefield, WV 24701, USA
}

\begin{abstract}
Cooperative adaptive cruise control (CACC) vehicles are intelligent vehicles that use vehicular ad hoc networks (VANETs) to share traffic information in real time. Previous studies have shown that CACC could have an impact on increasing highway capacities at high market penetration. Since reaching a high CACC market penetration level is not occurring in the near future, this study presents a progressive deployment approach that demonstrates to have a great potential of reducing traffic congestions at low CACC penetration levels. Using a previously developed microscopic traffic simulation model of a freeway with an on-ramp - created to induce perturbations and trigger stop-and-go traffic, the CACC system's effect on the traffic performance is studied. The results show significance and indicate the potential of CACC systems to improve traffic characteristics which can be used to reduce traffic congestion. The study shows that the impact of CACC is positive and not only limited to a high market penetration. By giving CACC vehicles priority access to high-occupancy vehicle (HOV) lanes, the highway capacity could be significantly improved with a CACC penetration as low as $20 \%$.
\end{abstract}

Keywords: Intelligent transportation systems, telematics, vehicular adhoc networks, cooperative adaptive cruise control, traffic simulation, agent-based traffic simulation.

\section{Introduction}

During the last decades, the United States is constantly facing the problem of traffic congestion and its related negative effects pertaining to safety, loss of productivity, wear and tear on vehicles and many others. The expectations of the use of telematics to solve this problem are high, since this technology has shown a possibility of increasing the highway capacity and the safety if widely adopted ${ }^{[1]}$

An intelligent transportation systems (ITS) system that is gaining popularity is cooperative adaptive cruise control (CACC). CACC vehicles are intelligent vehicles that have the ability of sharing traffic information via vehicular adhoc networks (VANETs) or wireless technology as well as regular radars (refer to Fig. 1). CACC is a new and further development of the adaptive cruise control (ACC) technology by having faster and more accurate real time information sharing among the equipped vehicles. Research has shown that CACC has a potential of improving the traffic dynamics (flow, density, speed variation, average speed, etc.) and safety if widely adopted (related literature covered in the next section). However, reaching a high CACC market penetration level is not occurring in the near future. In addition, the literature pertaining to the impact of CACC on traffic dynamics is lacking because the technology is still fairly new (no CACC equipped vehicles exist in the market at this point).

In order to address this topic, the CACC systems have to be explored thoroughly by studying how the drivers use the systems, how the equipped vehicles communicate and interact with each other, and most importantly, how the CACC systems impact certain traffic performance metrics at low penetration levels. Thus, this

Manuscript received September 10, 2012; revised April 25, 2013 study presents a progressive deployment approach that demonstrates how CACC could have a positive impact on traffic dynamics with penetration levels as low as $20 \%$.

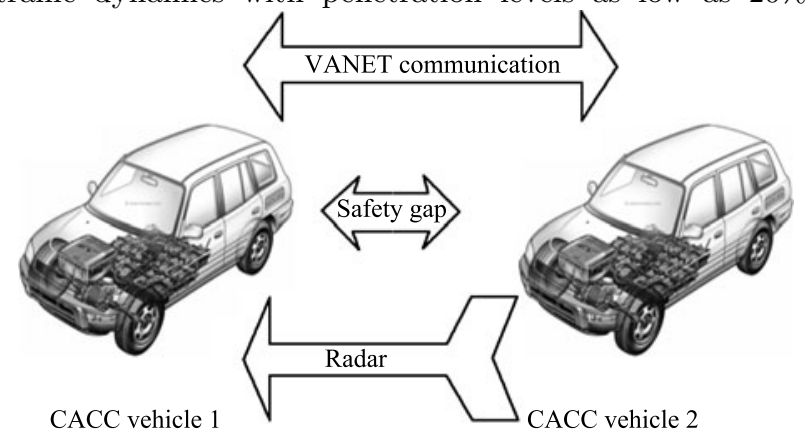

Fig. 1 CACC vehicle-to-vehicle communication using VANETs and radar

\section{Review of the literature}

ACC is a technology intended for the driver's comfort and safety but could have an impact on the overall traffic flow. References [2-4] studied the effects of ACC on the traffic performance concluding that the effect of ACC could be positive and negative at the same time. According to [5], ACC has no impact on the traffic performance if the level of ACC-equipped vehicles is low. The minimum time gap that can be achieved by ACC vehicles is 0.8 seconds $^{[6]}$. Reference [2] concluded in their study that with a relatively high penetration of ACC (more than $60 \%$ penetration), and even under the most advantageous conditions, the ACC system can only have a slight impact on highway capacity and performance.

Unlike ACC, the literature on CACC systems is still very premature. There are still no CACC design standards on 
how the technology operates. Thus, one area of research focuses on creating CACC designs frameworks, aiming to standardize and optimize the use of the technology. Several studies like [7-10] examined CACC designs and architectures, but most of the studies did not explore the traffic flow effects of CACC quantitatively in terms of throughput, capacity, and congestion reduction.

As it is expected in the near future that many (or most) of the vehicles will be fully automated, [11] studied a way to optimize the movements of the CACC vehicles through intersections to avoid collisions and minimize intersection delays.

One of the very few studies that targeted this paper's area of research was [2], which identified that CACC vehicles enable closer vehicle following (time gap as low as $0.5 \mathrm{~s}$ ) and concluded that the CACC technology has the potential to significantly increase the highway capacity — potentially doubling the capacity at a high market penetration. Another important finding of this study is that the capacity effect is very sensitive to the market penetration based on the fact that the reduced time gaps are only achievable between pairs of vehicles that are equipped with the CACC technology. However, the study simulated only a single-lane (meaning no overtaking is possible between vehicles). Even more, the study did not have trucks implemented in the model which could have a major effect on creating shockwaves on the freeway. Another equally important study was [1], which focused on the impact of CACC equipped vehicles on the traffic flow performance. The study revealed that CACC indeed shows a potential positive impact on the traffic throughput. In addition, CACC seems to increase highway capacity near a lane drop (bottleneck scenario). Furthermore, the impact of a dedicated CACC lane (i.e., a lane strictly operated by CACC-equipped vehicles) was studied, and it was shown that with a low CACC penetration $(<40 \%)$, the effect might lead to a degradation of traffic performance. Although the contribution of both studies $^{[1,2]}$ were beneficial to the CACC literature, both studies did not take into account modeling a CACC special lane that allows manual non-CACC vehicles to operate on. We believe that this approach could have a potential in using the advantage of CACC equipped vehicles being grouped together, without wasting lane capacity like in the case of having a CACC dedicated lane, under low CACC market penetration levels.

A stability analysis of a macroscopic simulation model conducted by [12] showed that the CACC system improves the dynamic equilibrium capacity over the ACC system. In a different study, [13] explored the effect of CACC on traffic dynamics on a multilane freeway (with the existence of a ramp) and showed that CACC indeed has a positive impact proportional to the CACC market penetration and the density of the traffic. The simulation model used in that study along with the proposed CACC algorithms were thoroughly discussed and validated in a separate study by [14]. Another study by [15] showed the effect of CACC to annul stop-and-go traffic in different traffic conditions. All these studies agreed that there is a need for a progressive deployment strategy for CACC since at penetration levels lower than $40 \%$ CACC, no significant impact was observed on the traffic flow.

\section{Simulation model details}

In order to study the impact of CACC on the traffic dynamics (particularly on traffic flow, average speed, and travel time spent), microscopic simulation is a suitable approach to study the individual behavior of every equipped vehicle (agent) and predict their overall impact on the whole system. Thus, a stochastic microscopic traffic simulation model F.A.S.T. (flexible agent-based simulator of traffic) was developed.

The object-oriented model developed using Javaß, is an expansion of a preexisting open-source microscopic model originally developed by [16]. Some of the most important additions to the original model are: increasing the highway distance from $2.5 \mathrm{~km}$ to $6 \mathrm{~km}$, adding two additional lanes (F.A.S.T. has 4-lanes), collecting microscopic properties as well as macroscopic properties, and most importantly modeling the behavior of CACC vehicles, with or without a priority access to high-occupancy vehicle (HOV) lanes referred to in this study as special HOV lanes (i.e., CACC accessible HOV lanes). Furthermore, additional randomness is added to the original model by manipulating the key variables of the car-following models and making them more stochastic. Note that Treiber's study focused on the ACC systems and their impact on the highway capacity while this study focuses on the CACC systems and their impact on traffic performance.

F.A.S.T. consists of a $6 \mathrm{~km}$ U-shaped freeway having four lanes, where ongoing traffic flows counterclockwise (shown in Fig. 2). Vehicles enter the system at different speeds and at a user-specified arrival rate, and exit after traveling the $6 \mathrm{~km}$ distance. There are two types of agents in F.A.S.T.: cars and trucks. The standard dimension of cars (whether CACC equipped or not) is $4 \times 2 \mathrm{~m}^{2}$ and for trucks is $6 \times 2 \mathrm{~m}^{2}$ (considering mid-sized trucks only).

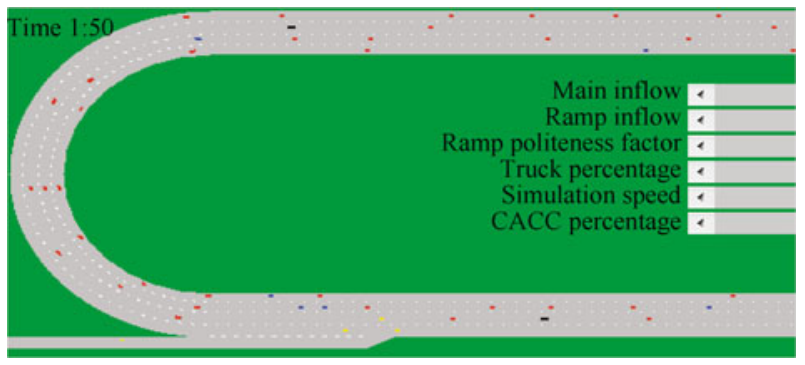

Fig. 2 Snapshot of the Java-based simulation model F.A.S.T

In order to generate a sufficient speed perturbation to our model, an on-ramp is added to the model, where the entering agents (all manual vehicles) may come to a full stop if there is not enough gap for them to enter the lane adjacent to the ramp. The 200 meters long ramp serves as a generator of perturbations that impact the traffic performance negatively by creating inhomogeneity in the speeds of the operating agents. A constant arrival rate of $500 \mathrm{veh} / \mathrm{h}$ will be entering the freeway from the on-ramp. The red agents are the non-CACC equipped vehicles, the black agents are the trucks, the yellow agents are the vehicles flowing from 
the ramp, and the blue agents are the CACC equipped vehicles.

Finally, all the vehicles in F.A.S.T. (CACC and manual) have different desired maximum speeds decided randomly following a uniform distribution between 100 and $120 \mathrm{~km} / \mathrm{h}$ for cars, and between 90 and $110 \mathrm{~km} / \mathrm{h}$ for trucks, considering that the speed limit imposed on the highway studied is $100 \mathrm{~km} / \mathrm{h}(\approx 60 \mathrm{mph})$. Drivers tend to go at increasing speeds whenever the roadway geometric characteristics are fine, regardless of the posted speed limit ${ }^{[17]}$. The algorithms used in F.A.S.T. were explained and validated in a previous study ${ }^{[14]}$. Note that in CACC, the driver retains full control of the vehicle at all times.

\section{Model validity}

In order to verify and validate the calibration of the proposed traffic model, the traffic data generated by the model was compared to historical data taken from the Highway Capacity Manual (HCM). According to the HCM, at a speed limit of $60 \mathrm{mph}$, the average flow rate in a multilane highway is around $2200 \mathrm{veh} / \mathrm{h} / \mathrm{ln}$ (refer to Table 1 ).

Table 1 Flow rate according to speed limits (source: Highway Capacity Manual, December 2000

\begin{tabular}{cc}
\hline Speed limit $(\mathrm{mph})$ & Flow rate $(\mathrm{veh} / \mathrm{h} / \mathrm{ln})$ \\
\hline 60 & 2200 \\
55 & 2100 \\
50 & 2000 \\
45 & 1900 \\
\hline
\end{tabular}

The model was simulated at an arrival rate of 8500 vehicles/h (for four lanes) having only manual vehicles operating on the $6 \mathrm{~km}$ highway with a speed limit of $60 \mathrm{mph}$ (or $100 \mathrm{~km} / \mathrm{h})$.

There were no trucks, no CACC vehicles, and no vehicles flowing from the on-ramp. Note that vehicles tend to go over the speed limit following a uniform distribution between $100 \mathrm{~km} / \mathrm{h}$ and $120 \mathrm{~km} / \mathrm{h}$.

It was observed that $8500 \mathrm{veh} / \mathrm{h}$ was the highest achievable arrival rate in order to keep a steady flow of traffic. After running the simulation model for 30 replications, 90 minutes each, the average flow rate of the simulation was $8425.20 \mathrm{veh} / \mathrm{h}$ (per four lanes) equivalent to $2106.3 \mathrm{veh} / \mathrm{h} / \mathrm{ln}$. This is a very close flow rate to the one obtained from the HCM.

Another way of validating the proposed model was to compare the speed-flow curve for multilane highway sections according to historical data taken from the HCM. Fig. 3 shows the speed-flow relation at different speeds. The free-flow speed - the speed that a driver would travel if there were no congestion or other adverse conditions ahead, tends to be stable until the flow rate reaches a specific level where it starts dropping.

A similar pattern was observed in the proposed model after running simulations with increasing arrival rates until reaching $8500 \mathrm{veh} / \mathrm{h}$ (or $2125 \mathrm{veh} / \mathrm{h} / \mathrm{ln}$ ) and taking a snapshot of the output at a time without any congestion. Fig. 4 shows the speed-flow curve generated from the proposed model (scattered line) compared to the HCM curve (solid line).

The curves in Fig. 4 (compared to Fig. 3) are very similar in behavior and follow the same pattern. As observed, after reaching a certain maximum capacity, the speed starts to drop.

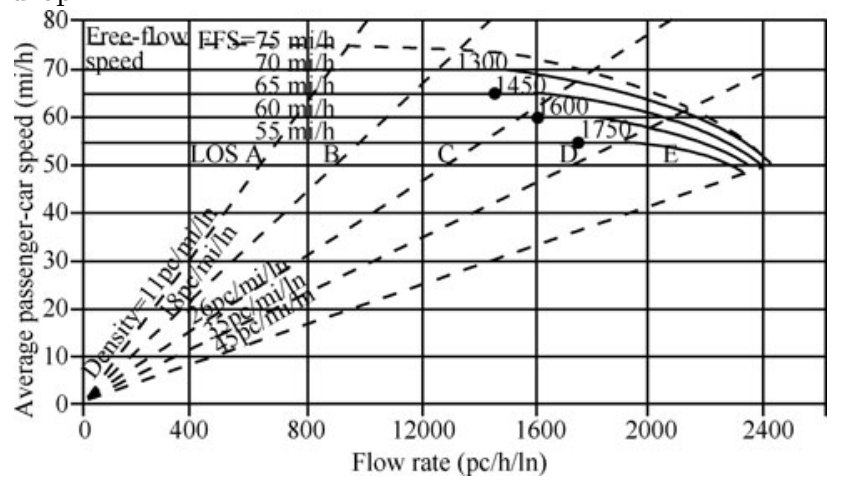

Fig. 3 Speed-flow curves for multilane highway sections (source: Highway Capacity Manual, December 2000, Exhibit 21-3, pp. 214

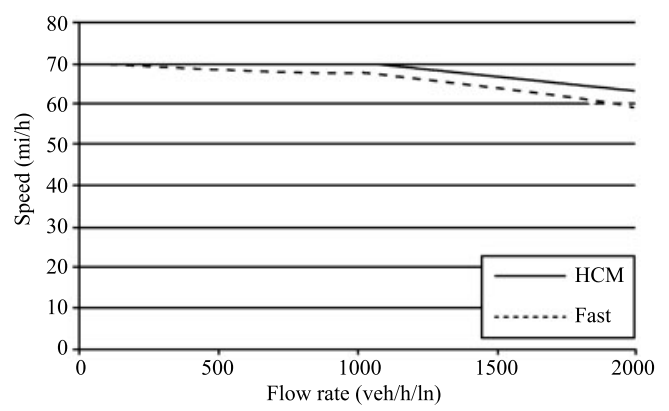

Fig. 4 Speed-flow curves in the simulation model

\section{Simulation experiments and results}

The traffic simulation F.A.S.T. was used to examine the significance of the impact of CACC on the traffic dynamics and particularly on the traffic flow, the average speed and the travel time spent.

\subsection{Simulation setup}

Following the previous literature about ACC and CACC designs ${ }^{[9,18,19]}$ and the algorithms from [14], the timegap setting of the CACC is set to $0.5 \mathrm{~s}$ if following a CACC vehicle. In other words, when both vehicles are CACC equipped, their time gap setting is set to $0.5 \mathrm{~s}$ where both vehicles are connected via wireless communication. When a CACC equipped vehicle is following a truck or a non-CACC equipped vehicle, the time gap is set to a headway gap uniformly distributed between $0.8 \mathrm{~s}$ (younger/aggressive drivers) and $1.0 \mathrm{~s}$ (older/considerate drivers) - acting in the same manner as an ACC vehicle and relying only on its radar but without establishing any wireless communication. In the case of a non-CACC vehicle (i.e., manual) following another CACC vehicle or non-CACC vehicle following another non-CACC vehicle (no wireless communication is established; no radar), the time gap setting is between $1.0 \mathrm{~s}$ and $1.8 \mathrm{~s}$ (uniform distribution) 
which is a commonly observed headway gap reported in previous studies ${ }^{[20-22]}$

For traffic generation, a previously user defined incoming traffic is divided randomly between the four lanes. A high traffic scenario (saturated/oversaturated) can lead to congestion on the freeway, while a low traffic scenario (under saturated) can result in a free flow of vehicles. The base case (initial state of the system), is having no CACC equipped vehicles operating with an arrival traffic rate of $4000 \mathrm{veh} / \mathrm{h}$ (on four lanes). The penetration rate of CACC systems varied between $0 \% \mathrm{CACC}$ and $20 \%$ CACC. The arrival rate varied between five scenarios: $4000 \mathrm{veh} / \mathrm{h}, 5000 \mathrm{veh} / \mathrm{h}$, $6000 \mathrm{veh} / \mathrm{h}, 7000 \mathrm{veh} / \mathrm{h}$, and $8000 \mathrm{veh} / \mathrm{h}$. The percentage of trucks in the system is fixed at $10 \%$. The $4000 \mathrm{veh} / \mathrm{h}$ and $5000 \mathrm{veh} / \mathrm{h}$ were considered a low traffic density scenario (no traffic jams but with some oscillations observed resulting from the ramp) while the other scenarios had more congestions especially the $7000 \mathrm{veh} / \mathrm{h}$ and the $8000 \mathrm{veh} / \mathrm{h}$ (where severe traffic jams were observed). At $6000 \mathrm{veh} / \mathrm{h}$, a stop-and-go traffic is observed with minor traffic congestion occurrences. The model was run for warm-up periods of five minutes in every replication as the system is initially empty (no vehicles/agents on the highway). From the observation of the model animation as well as the output, 5 minutes is sufficient to reach conditions that are typical of normal running traffic conditions in order to obtain more accurate results.

Since previous studies (refer to the literature review section) have already showed that CACC has indeed a significant positive impact on the traffic dynamics at high market penetration levels ( $>40 \%$ CACC), the experiments conducted in this paper relaxed that constraint by limiting the number of CACC penetration to $20 \%$ and giving the CACC vehicles priority access to special HOV lanes.

The simulation model was extended allowing CACC equipped vehicles to operate solely on special lanes or more accurately special HOV lanes. This approach enables the researchers to study the effect of a low CACC penetration rate on the traffic dynamics by giving $\mathrm{CACC}$ vehicles priority access to special lanes, in this case HOV lanes, allowing the CACC vehicles to operate closer to each other. This is an initial stage before a higher penetration level of CACC is reached (i.e., market penetration of $40 \%$ or more) where the CACC vehicles could be scattered on all the lanes and still improve the traffic performance. A small percentage of $10 \%$ manual vehicles were allowed to use the special HOV lanes considering that those vehicles have two or more passengers onboard. In addition, CACC equipped vehicles are initially created on the special HOV lane that we will refer to as lane 3. Fig. 5 shows the different lanes with their relative numbers. CACC vehicles do not change lanes at any part or time in the system.

Direction of the vehicles

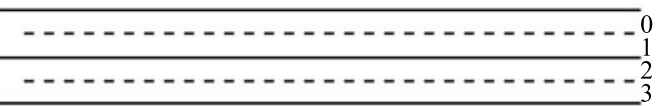

Fig. 5 Different lanes in the simulation model grouped by number
Vehicles that are not CACC equipped are uniformly created on the four lanes in the system. However, lane 3 has a much smaller ratio of non-CACC to CACC than the other lanes because $20 \%$ of the overall vehicles are CACC equipped and are located on lane 3 . In addition, non-CACC equipped vehicles that are initialized on lanes 0,1 , or 2 are allowed to switch lanes to lane 3 but only on a $10 \%$ probability (i.e., $10 \%$ of the non-CACC vehicles are allowed to switch the special HOV lane). Non-CACC equipped vehicles operating on lane 3 are allowed to change lanes to other lanes only if their incentive criterion was met (refer to the MOBIL lane change model by [23]. Trucks are allowed to operate on $\mathrm{HOV}$ lanes following the same rules that impact the non-CACC equipped cars. A snapshot taken in this phase is shown in Fig. 6 where the formation of platoons (groups of blue CACC vehicles) on the special HOV lane is observed.

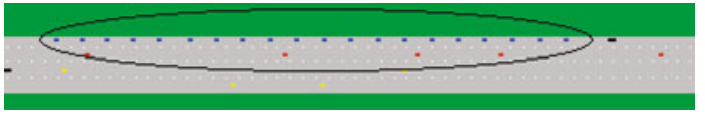

Fig. 6 Snapshot of F.A.S.T. with special HOV lanes

A comparison was made between scenarios of low CACC penetration levels of $20 \%$ with priority access to the special HOV lanes (referred to as $20 \%$ CACC HOV scenario), scenarios without CACC vehicles operating on the highways (without special lanes; referred to as $0 \%$ CACC), and scenarios with $20 \%$ CACC scattered on all the lanes without any special lanes (referred to as $20 \%$ CACC scattered). Section 5.2 covered these experiments with a ramp creating perturbations in the traffic flow. Section 5.3 covered the same experiments but without the ramp, having the same three scenarios in comparison $(0 \%$ CACC vs. $20 \%$ CACC HOV vs. $20 \%$ CACC scattered). Higher arrival rates of $7000 \mathrm{veh} / \mathrm{h}, 8000 \mathrm{veh} / \mathrm{h}, 9000 \mathrm{veh} / \mathrm{h}$, and $10000 \mathrm{veh} / \mathrm{h}$ were modeled in the scenario without a ramp.

The vehicles were allowed to overtake and weave. All the experiments had the same previous base assumptions:

1) Good weather (no rain, snow, fog, etc.).

2) Good pavement conditions.

3) No impediments to traffic flow.

4) Accidents and failures in the operation of the sensors and communication of CACC design equipment were not taken into consideration.

\subsection{Experiments with a ramp}

To ensure statistical validity, 30 stochastically independent simulations were performed for each selected scenario. Having a total of 15 scenarios (three different penetration rates and five different arrival rates), the simulations were run for 90 minutes per each replication. A constant arrival rate of $500 \mathrm{veh} / \mathrm{h}$ was set for the vehicles entering the freeway from the on-ramp to create perturbations in the traffic flow. Three performance metrics were collected and analyzed: traffic flow rate, average speed of the vehicles, and the travel time spent. The flow rate was collected by getting the throughput of the agents passing through two fixed points located at 100 meters before the ramp. The average speed (time mean speed) was collected by averaging the speed of all the agents passing through a fixed point 
located at 100 meters before the ramp acting as a loop detector. The travel time spent was collected by averaging the total time spent by every agent in the system (time of exit minus time of entry).

\subsubsection{Flow rate analysis (with ramp)}

Fig. 7 shows the rate of flow in different scenarios comparing 20\% CACC penetration operating on special $\mathrm{HOV}$ lanes, 20\% CACC scattered on all the lanes, and the base case of $0 \%$ CACC penetration.

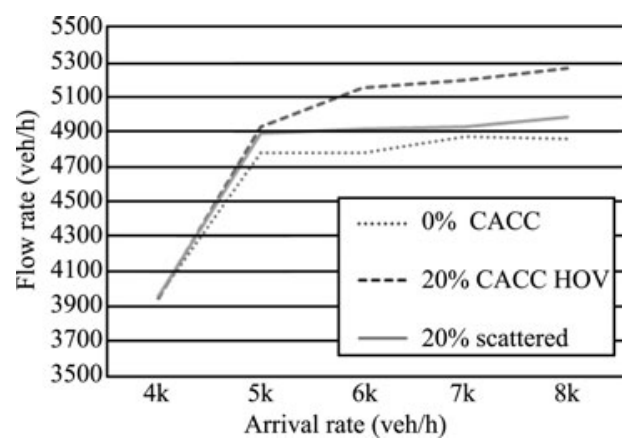

Fig. 7 Flow rate analysis (with ramp)

Observing Fig. 7, the scenario of $20 \%$ CACC HOV shows superiority over the other cases with a traffic flow rate proportional to the increasing arrival rate of the vehicles. Since the flow rate in all the three scenarios compared was somehow close, ANOVA test was conducted to identify the statistical significance of CACC in the different scenarios studied, followed by Dunnett T3 Post hoc analysis to find relations and patterns among the different scenarios. For the $4000 \mathrm{veh} / \mathrm{h}$ arrival rate scenario, ANOVA showed no significance between all the three scenarios. In the $5000 \mathrm{veh} / \mathrm{h}$ scenario, significance was found in both $20 \%$ CACC penetration (scattered and HOV cases) compared to the base case of $0 \%$ CACC. However, the Dunnett T3 test showed no significance between the two $20 \%$ CACC penetration scenarios resulting in a $p>0.05$. At $6000 \mathrm{veh} / \mathrm{h}$, a statistical significance between the two $20 \%$ CACC penetration scenarios was observed (showing the superiority of the HOV scenario). In the $7000 \mathrm{veh} / \mathrm{h}$ scenario, no significance was found between the base case of no CACC penetration and the $20 \%$ CACC scattered scenario. However, the $20 \%$ CACC HOV outperformed the base case scenario significantly. In the $8000 \mathrm{veh} / \mathrm{h}$ scenario, the behavior was somewhat similar to the previous case of $7000 \mathrm{veh} / \mathrm{h}$ where the $20 \%$ CACC HOV scenario outperformed the two other scenarios significantly, demonstrating the superiority of the $20 \%$ CACC HOV scenario over the other scenarios.

Thus, the results confirmed that placing the CACC vehicles on special HOV lanes, at a low market penetration of $20 \%$, has a statistically significant positive effect on the traffic flow. This effect is highly observed when the traffic is saturated (i.e., arrival rate of $6000 \mathrm{veh} / \mathrm{h}$ and more) because at free-flow conditions, all the scenarios have a freeflow traffic that is able to reach the maximum capacity of the freeway.

\subsubsection{Time spent analysis (with ramp)}

Additionally, in Fig. 8, a comparison of the average time spent in the system by the vehicles was collected. The three cases of $0 \%$ CACC, $20 \%$ CACC scattered, and $20 \%$ CACC $\mathrm{HOV}$ were compared.

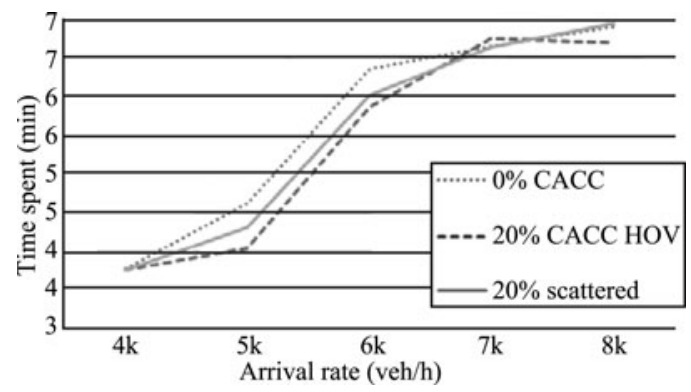

Fig. 8 Travel time spent analysis (with ramp)

An S-shape function was observed with no direct relation between the CACC penetration level and the time spent at $4000 \mathrm{veh} / \mathrm{h}$ arrival rate. ANOVA was conducted resulting in $\mathrm{Sig}=0.531$ (showing that the difference is not statistically significant). At $5000 \mathrm{veh} / \mathrm{h}$ arrival rate, the $20 \%$ CACC scattered had a slight significance compared to the $0 \%$ CACC $(p=0.049)$. The $20 \%$ CACC HOV showed statistical significance compared to the base case $(p=0.00)$ and the difference between the $20 \%$ CACC scattered and the $20 \%$ CACC HOV was statistically significant. In the $6000 \mathrm{veh} / \mathrm{h}$ arrival rate, both scenarios with the $20 \%$ CACC outperformed the base case significantly, but the difference between them (scattered vs. HOV) was not statistically significant. At $7000 \mathrm{veh} / \mathrm{h}$ and $8000 \mathrm{veh} / \mathrm{h}$, ANOVA showed no significance in the results ( $\mathrm{Sig}=0.709$ for the $7000 \mathrm{veh} / \mathrm{h}$ and Sig $=0.454$ for the $8000 \mathrm{veh} / \mathrm{h}$ ). Thus, it is concluded that with the presence of a ramp, the $20 \%$ CACC HOV scenario performed slightly better than the two other scenarios where vehicles spent less time in the system at low to medium arrival rates (lower than $7000 \mathrm{veh} / \mathrm{h}$ ). At high arrival rates, the results were somehow similar (no statistical difference) between the three scenarios studied. However, this does not mean that the $20 \%$ CACC HOV performed poorly. Since the flow rate increases proportional to the increasing arrival rate, and with a decreasing average speed ${ }^{1}$ in the $20 \%$ CACC HOV (explained thoroughly in Section 5.2 .3 ), this proves that the density is increasing (from the simple traffic flow formula $q=k \times v$. If $q$ or flow is increasing and $v$ or speed is decreasing, $k$ has to be increasing). Therefore, the density in the $20 \%$ CACC HOV is higher than the density of the other scenarios (in which the flow was lower and the next section shows that the average speed was higher). Thus, with a higher density, it would not be accurate to compare the travel time spent between the scenarios as the densities are completely different.

\subsubsection{Average speed analysis (with ramp)}

The impact of CACC on the highway average speed in the three scenarios: $20 \%$ CACC scattered, 20\% CACC HOV, and $0 \%$ CACC, was analyzed using one-way ANOVA. Fig. 9

\footnotetext{
${ }^{1}$ Note that although the traffic flow formula used applies only to the space mean speed (and not the time mean speed we are using in this study), the time mean speed is always higher than the space mean speed. Therefore the space mean speed will follow the same decreasing behavior as the time mean speed validating the assumption made.
} 
shows the average speed at different arrival rates of vehicles. At $4000 \mathrm{veh} / \mathrm{h}$, the results of ANOVA did not show any statistical significance $(p=0.185)$ between the means of the three scenarios.

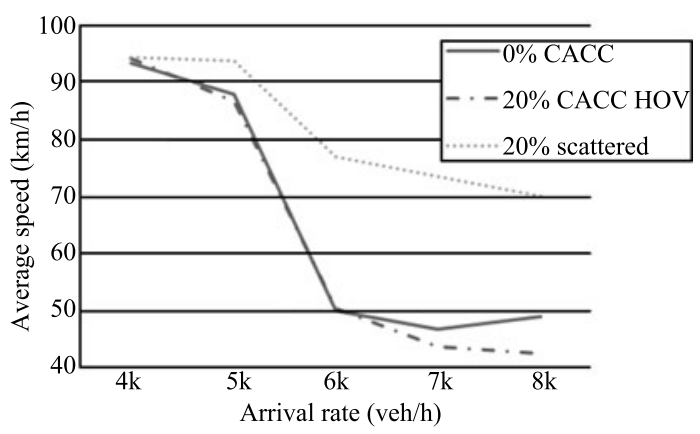

Fig. 9 Average speed analysis (with ramp)

At $5000 \mathrm{veh} / \mathrm{h}$, the results were not significant between the $20 \%$ CACC HOV and the $0 \%$ CACC base case. However, statistical significance was found between the $20 \%$ CACC scattered scenario and the base case. Also, statistical significance was found between the $20 \%$ CACC scattered scenario and the $20 \%$ CACC HOV scenario, showing that the $20 \%$ CACC scattered case had a higher average speed. At $6000 \mathrm{veh} / \mathrm{h}, 7000 \mathrm{veh} / \mathrm{h}$, and $8000 \mathrm{veh} / \mathrm{h}$, the results followed the same behavior. When comparing the $20 \%$ CACC HOV scenario with the base case, no statistical significance was found. When comparing the $20 \%$ CACC HOV scenario with the $20 \%$ CACC scattered scenario, statistical significance was found where the $20 \%$ CACC scattered scenario resulted in superior average speed. In the case of $20 \%$ $\mathrm{CACC} \mathrm{HOV}$, the average speed collected was lower than the other two cases studied. However, the flow in this case outperformed the other two cases. The reason for the lower average speed in the $20 \% \mathrm{CACC} \mathrm{HOV}$ scenario is that at a higher arrival rate and with sufficient CACC penetration, the speed is being divided among the operating vehicles (CACC and non-CACC equipped). This shows that the accelerations and decelerations of the vehicles became smaller resulting in a smoother flow of traffic because the CACC vehicles (that are supposed to reduce the standard deviation of the speed) are placed only on one lane - lane 3, out of four available lanes. Thus, the reduction of speed was due to a slower but steady traffic where traffic jams did not occur. This results in a higher rate of flow and a lower overall average speed. A study by [24] explored such behavior by studying the effects of an on-ramp on the traffic flow. The study suggested an explanation for this hysteretic effect effect of a system that has a memory is not felt at the same instant. Reference [24] simulated freeways with on-ramps with a fluid-dynamic traffic model and explained how the average vehicle speed will adapt to an equilibrium speed, which monotonically decreases with the growing density of the traffic.

\subsection{Experiments without a ramp}

In this phase, experiments were without an on-ramp to model the effect of "natural" shockwaves (shockwaves resulting only from vehicles' decelerations) on traffic perfor- mance without the impact of vehicles flowing from an onramp creating perturbations in the flow. The same three performance metrics from Section 5.2 were used (and collected in the same way). In these experiments, higher arrival rates were chosen because of the absence of the onramp that plays a major role in creating perturbations that ultimately leads to traffic congestions. The arrival rates of $7000 \mathrm{veh} / \mathrm{h}$ and $8000 \mathrm{veh} / \mathrm{h}$ are cases where no traffic congestions occurred (low traffic hours). The arrival rates of $9000 \mathrm{veh} / \mathrm{h}$ and $10000 \mathrm{veh} / \mathrm{h}$ are cases where traffic congestions were observed (high traffic hours).

5.3.1 Flow rate analysis (without a ramp)

Fig. 10 shows the rate of flow between the three different cases studied and different arrival rates.

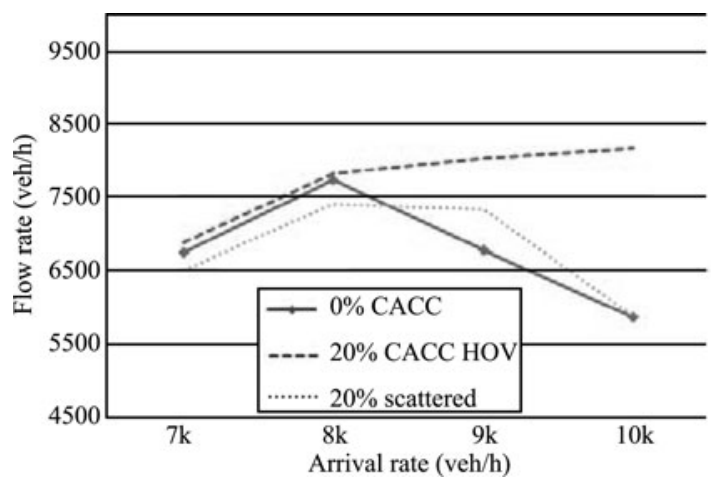

Fig. 10 Flow rate analysis (without a ramp)

As observed in Fig. 10, the more vehicles arrive to the system, the flow increases until reaching a critical point where it starts declining. At $0 \% \mathrm{CACC}$ the critical point is at $8000 \mathrm{veh} / \mathrm{h}$ where after that point the flow starts degrading. Note that when validating the model, the arrival rate of $8500 \mathrm{veh} / \mathrm{h}$ was the critical point before the flow started to degrade. However, the difference here is that trucks were added. Trucks create perturbations to the traffic and reduce the rate of flow by adding more perturbations to it. At $20 \%$ CACC scattered, the flow rate was slightly better where after $8000 \mathrm{veh} / \mathrm{h}$, the flow rate stayed constant until $9000 \mathrm{veh} / \mathrm{h}$; but then at $10000 \mathrm{veh} / \mathrm{h}$, it degraded. At $20 \%$ CACC HOV, the flow rate kept increasing exponentially beyond the critical point of $8000 \mathrm{veh} / \mathrm{h}$ and even beyond the highest arrival rate of $10000 \mathrm{veh} / \mathrm{h}$.

To confirm the stated observations, one-way ANOVA test followed by Dunnett T3 post hoc tests were conducted to evaluate the difference between the three cases studied. At $7000 \mathrm{veh} / \mathrm{h}$, there was no statistical significance between $0 \%$ CACC and 20\% CACC HOV. There was a slight statistical significance between 0\% CACC and 20\% CACC scattered where the flow of $0 \%$ CACC was higher. At $8000 \mathrm{veh} / \mathrm{h}$, a slightly different behavior was observed. There was no statistical significance between the $0 \% \mathrm{CACC}$ and $20 \% \mathrm{CACC}$ $\mathrm{HOV}$ cases, nor between the $0 \% \mathrm{CACC}$ and $20 \% \mathrm{CACC}$ scattered. Statistical significance was found between $20 \%$ CACC HOV and 20\% CACC scattered. This shows that in low traffic densities, the effect of CACC is minimal and most of the time non-significant. Note that in the previous two scenarios, no congestion was detected. At $9000 \mathrm{veh} / \mathrm{h}$, there was an improvement observed between the $0 \% \mathrm{CACC}$ 
case and the $20 \%$ CACC scattered case. However, the improvement was not statistically significant. On the other hand, the improvement between the $0 \%$ CACC base case and the $20 \%$ CACC HOV case was significant (with $p=$ $0)$. Note that in this case, some congestions were observed but not in all the replications. Finally, at $10000 \mathrm{veh} / \mathrm{h}$ arrival rate, congestions occurred at some point in all the cases. Less congestion was observed in the $20 \%$ CACC HOV. There was no significance between $0 \%$ CACC and $20 \%$ CACC scattered cases. However, there was a statistical significance between the base case of $0 \% \mathrm{CACC}$ and the case of $20 \%$ CACC HOV that showed a major improvement in the flow rate.

It is concluded from the results that the CACC increases the highway capacity even at low penetration levels if placed on special HOV lanes. However, this effect is highly dependent on the arrival rate of the vehicles. It is extremely important here to mention that the system reaches its maximum capacity in the $0 \%$ CACC scenario and the $20 \%$ CACC scattered scenario which was around $8000 \mathrm{veh} / \mathrm{h}$ (per four lanes) at the moderate arrival rate scenario of $8000 \mathrm{veh} / \mathrm{h}$. However, the more we increased the arrival rate above that limit to $9000 \mathrm{veh} / \mathrm{h}$ and $10000 \mathrm{veh} / \mathrm{h}$, the queues of the vehicles stretched until reaching the system's entrance and no additional agents were able to enter the system. As this shows the definitive superiority of the $20 \%$ CACC HOV scenario, the ANOVA comparisons conducted at these conditions are not considered very accurate because at $9000 \mathrm{veh} / \mathrm{h}$ and $10000 \mathrm{veh} / \mathrm{h}$, the system was oversaturated and no agents were entering the system resulting in extenuating a deadlock that was ignored in the analysis.

\subsubsection{Time spent analysis (without a ramp)}

Additionally, in Fig. 11, a comparison of the average time spent in the system by the vehicles was collected. The same three cases of $0 \%$ CACC, $20 \%$ CACC scattered on all the lanes, and 20\% CACC operating solely on the special HOV lanes were compared.

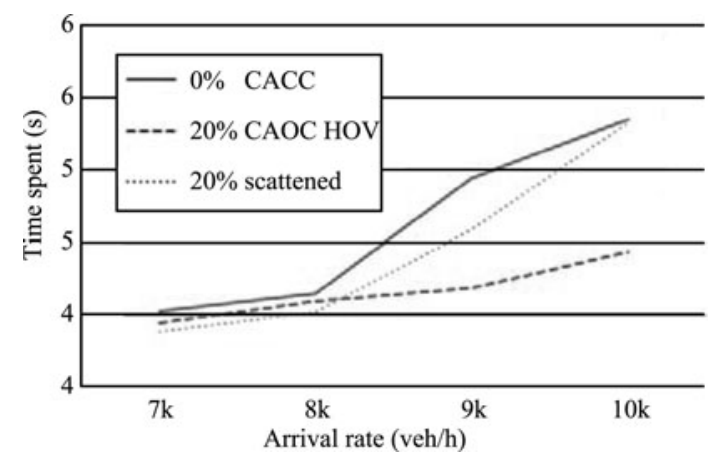

Fig. 11 Time spent analysis (without a ramp)

The difference in the results is observed at arrival rates higher than $8000 \mathrm{veh} / \mathrm{h}$ where the $0 \%$ CACC vehicles took longer to travel through the system, and the $20 \%$ CACC HOV vehicles evidently performed better than the other two cases. To validate these observations, ANOVA was conducted. At $7000 \mathrm{veh} / \mathrm{h}$, the difference between the $20 \%$ CACC HOV and the two other scenarios was not significant. At $8000 \mathrm{veh} / \mathrm{h}$, ANOVA showed also no statistical significance. At $9000 \mathrm{veh} / \mathrm{h}$ no significance was observed between the $20 \%$ CACC scattered and the base case. The results of the $20 \%$ CACC HOV showed statistical significance and outperformed the two other scenarios. The exact same behavior was observed at a $10000 \mathrm{veh} / \mathrm{h}$ arrival rate, showing the positive impact of $\mathrm{CACC}$ in reducing the travel time for the vehicles but only in high arrival rates when traffic is saturated.

\subsubsection{Average speed analysis (without a ramp)}

The average speed was also analyzed. The $20 \%$ CACC HOV showed an overall better performance than the other two cases as shown in Fig. 12.

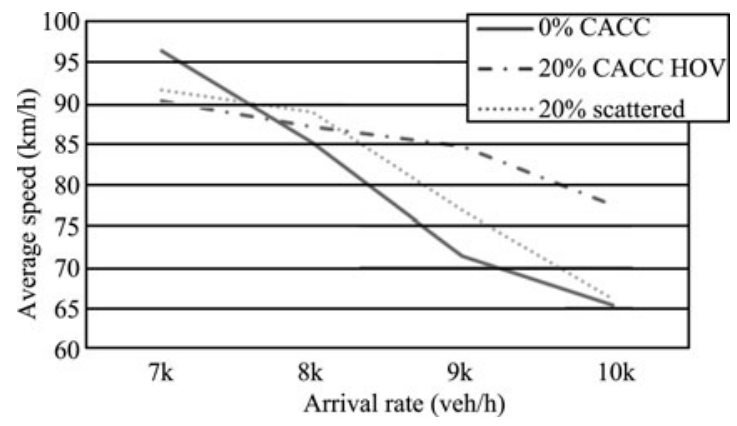

Fig. 12 Average speed analysis (without a ramp)

ANOVA test was conducted to evaluate the significance of the results and compare the three different cases correctly. In the $7000 \mathrm{veh} / \mathrm{h}$ and $8000 \mathrm{veh} / \mathrm{h}$ (low traffic hours), ANOVA showed no statistical significance between the results of the three cases studied. At $9000 \mathrm{veh} / \mathrm{h}$, significance has been found between $0 \%$ CACC and 20\% CACC HOV case and between 20\% CACC HOV and 20\% CACC scattered. The results showed that the $20 \%$ CACC HOV outperformed the other cases. Although the results of the $20 \%$ CACC scattered looked better than the $0 \%$ CACC base case, the improvement was not statistically significant. At $10000 \mathrm{veh} / \mathrm{h}$, the behavior was similar to the previous scenario. There was no significance between the 0\% CACC base case and the $20 \%$ CACC scattered case. On the other hand, the $20 \%$ CACC HOV performed better than both scenarios with statistical significance. This validates the previous claim that at higher arrival rates, a low percentage of CACC equipped vehicles placed on special HOV lanes have a significant positive impact on the average speed. However, the CACC impact is highly dependent on the arrival rates of the vehicles.

It is worth mentioning that in Section 5.2.3, the average speed was lower although the flow was higher because of the hysteretic effects of the on-ramp that was present at that phase. The on-ramp played a major role in reducing the speed to an equilibrium speed that decreases inversely proportional to the growing density as explained in a study by ${ }^{[24]}$. In this phase, this effect was not observed because the on-ramp was not present.

\section{Conclusions}

In this paper, F.A.S.T, a microscopic traffic simulator that simulates the effect of cooperative adaptive cruise control (CACC) equipped vehicles on a four-lane freeway, was 
used to identify the impact of CACC on traffic dynamics in low CACC market penetration. CACC vehicles are not available in the car market at this point. It is assumed that their spreading will grow in the future. Since reaching a high CACC penetration level is not occurring in the near future, this study presented a progressive deployment approach that demonstrated to have a great potential of reducing traffic congestions at low CACC penetration levels by placing $\mathrm{CACC}$ vehicles on $\mathrm{HOV}$ lanes until reaching a 40\% CACC penetration level. At 40\% CACC and higher, previous related studies $^{[1,2,13]}$ showed the positive significance of CACC by simply scattering the vehicles on all the lanes of the freeway.

\section{Discussion and future work}

The public acceptability of the CACC technology is a major challenge facing the CACC studies. A study by [25] showed that most of the people would not feel comfortable driving at a headway gap of $0.5 \mathrm{~s}$ and considered it to be too close to the preceding vehicle. With the driver retaining full control of the vehicle at all times, we believe that the public acceptance of the CACC technology will only be a temporary challenge solved by its increasing usage and design standardization, just like any new breakthrough technology.

Perhaps the most important improvement needed in the proposed model, is for it to be extended to allow accidents to occur and to implement other obstacles on the roads (a blocked lane to create a bottleneck for example), forcing the vehicles to merge into a smaller number of lanes. Additional scenarios could be added to explore the impacts of CACC on the traffic dynamics more efficiently. Up to now, the CACC platoons once formed do not deal with the size of the platoons and the possibility of a vehicle attempting to join/exit the platoon (to take an exit for instance). Platooning algorithms could be explored and mixed with the existing CACC algorithm, in order to avoid possible problems resulting from inefficient platooning practices.

\section{References}

[1] B. Van Arem, C. J. G. Van Driel, R. Visser. The impact of cooperative adaptive cruise control on traffic-flow characteristics. IEEE Transactions on Intelligent Transportation Systems, vol. 7, no. 4, pp. 429-436, 2006.

[2] J. VanderWerf, S. Shladover, M. Miller, N. Kourja. Evaluation of the effects of adaptive cruise control systems on highway traffic flow capacity and implications for deployment of future automated systems. In Proceedings of the 81st Annual Meeting of the Transportation Research Board, pp. 78-84, 2002.

[3] P. J. Zwaneveld, B. V. Arem. Traffic Effects of Automated Vehicle Guidance System, A Literature Survey, TNO Inro, TNO-Report INRO-VVG, Delft, The Netherlands, 1997.

[4] A. E. Hoetink. Advanced Cruise Control en verkeersveiligheid, R-2003-24. Institute for Road Safety Research (SWOV), Leidschendam, The Netherlands, 2003.

[5] B. Van Arem, J. H. Hogema, M. J. W. A. Vanderschuren, C. H. Verheul. An Assessment of the Impact of Autonomous Intelligent Cruise Control, INRO-VVG, Report, Delft, The Netherlands, 1995.
[6] G. Marsden, M. McDonald, M. Brackstone. Towards an understanding of adaptive cruise control. Transportation Research Part C: Emerging Technologies, vol. 9, no. 1, pp. 33$51,2001$.

[7] S. E. Shladover. Effects of Cooperative Adaptive Cruise Control on Traffic Flow: Testing Drivers' Choices of Following Distances, California PATH Research Report, University of California, 2009.

[8] A. R. Girard, J. B. de Sousa, J. A. Misener, J. K. Hedrick. A control architecture for integrated cooperative cruise control and collision warning systems. In Proceedings of the 40th IEEE Conference on Decision and Control, IEEE, Orlando, USA, pp. 1491-1496, 2001.

[9] D. de Bruin, J. Kroon, R. van Klaveren, M. Nelisse. Design and test of a cooperative adaptive cruise control system. In Proceedings of IEEE Intelligent Vehicles Symposium, IEEE, Parma, Italy, pp. 392-396, 2004.

[10] J. A. Misener, A. R. Girard, M. C. Drew. Experimental implementation of cooperative adaptive cruise control. In Proceedings of the 9th World Congress on Intelligent Transport Systems, Chicago, Illinois, USA, pp. 8, 2002.

[11] I. Zohdy, R. K. Kamalanathsharma, H. Rakha. Intersection management for autonomous vehicles using cooperative adaptive cruise control systems. In Proceedings of the 92nd Annual Meeting of the Transportation Research Board, Washington, DC, USA, 2013.

[12] D. Ngoduy. Instability of cooperative adaptive cruise control traffic flow: A macroscopic approach. Communications in Nonlinear Science and Numerical Simulation, vol.18, no. 10, pp. 1838-3851, 2013.

[13] G. M. Arnaout, S. Bowling. Towards reducing traffic congestion using cooperative adaptive cruise control on a freeway with a ramp. Journal of Industrial Engineering and Management, vol. 4, no. 4, pp. 699-717, 2011.

[14] G. M. Arnaout, H. Arbabi, S. Bowling. An effective cooperative adaptive cruise control algorithm for intelligent transportation systems. Journal of IET Intelligent Transportation Systems, 2010. (Online first).

[15] J. Q. Ma, F. Zhou, M. J. Demetsky. Evaluating mobility and sustainability benefits of cooperative adaptive cruise control using agent-based modeling approach. In Proceedings of 2012 IEEE Systems and Information Design Symposium (SIEDS), IEEE, Charlottesville, VA, USA, pp. 74-78, 2012

[16] M. Treiber. Microsimulation of Road Traffic Flow, [Online], Available: http://www.traffic-simulation.de/, 2010.

[17] N. J. Garber, R. Gadirau. Speed Variance and Its Influence on Accidents, Washington. DC, USA: AAA Foundation for Traffic Safety, pp. 69, 1988.

[18] G. Naus, R. Vugts, J. Ploeg, R. van de Molengraft, M. Steinbuch. Cooperative adaptive cruise control, design and experiments. In Proceedings of 2010 American Control Conference, IEEE, Baltimore, MD, USA, pp. 6145-6150, 2010.

[19] A. Kesting, M. Treiber, M. Schönhof, F. Kranke, D. Helbing. Jam-avoiding adaptive cruise control (ACC) and its impact on traffic dynamics. Traffic and Granular Flow'05 (Part VI), Berlin, Heidelberg, Germany: Springer, pp. 633643, 2007.

[20] M. Treiber, A. Kesting, D. Helbing. Understanding widely scattered traffic flows, the capacity drop, and platoons as effects of variance-driven time gaps. Physical Review E, vol. 74, no. 1, pp. 016123, 2006.

[21] W. Knospe, L. Santen, A. Schadschneider, M. Schreckenberg. Towards a realistic microscopic description of highway traffic. Journal of Physics A: Mathematical and General, vol. 33, no. 48, pp. L477-L485, 2000 
[22] D. H. Ha, M. Aron, S. Cohen. Time headway variable and probabilistic modeling. Transportation Research Part C: Emerging Technologies, vol. 25, pp. 181-201, 2012.

[23] A. Kesting, M. Treiber, D. Helbing. General lane-changing model MOBIL for car-following models. Transportation Research Record: Journal of the Transportation Research Board, vol. 1999, pp. 86-94, 2007.

[24] H. Y. Lee, H. W. Lee, D. Kim. Origin of synchronized traffic flow on highways and its dynamic phase transitions. Physical Review Letters (American Physical Society), vol. 81, no. 5, pp. 1130-1133, 1998.

[25] S. E. Shladover, C. Nowakowski, D. Cody, F. Bu, J. $\mathrm{O}^{\prime}$ Connell, J. Spring, S. Dickey, D. Nelson. Effects of cooperative adaptive cruise control on traffic flow: testing drivers' choices of following distances, California PATH Research Report, Institute of Transportation Studies, University of California, Berkeley, 2009.

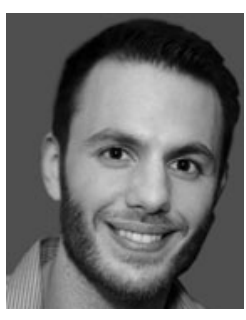

Georges M. Arnaout received his $\mathrm{Ph}$. D. degree in engineering management (concentration on simulation) from the Department of Engineering Management and Systems Engineering at Old Dominion University, USA. He received his B.Sc. degree in management information systems from Balamand University, Lebanon in 2006. He received his M.Sc. degree in computer science with an emphasis in information systems from Old Dominion University in 2008. He currently holds a position of a simulation engineer in Kiva Systems Incorporated, a subsidiary of Amazon.com. He has various papers published and/or submitted in the area of modeling and simulation.

His research interests include modeling and simulation. agentbased modeling of cooperative adaptive cruise control impact on freeway traffic flow, using modeling and simulation for decision making in complex environments. He is fond of topics relating to modeling and simulation including operations research, discreteevent, systems dynamics, and agent-based modeling.

E-mail: Georgearnaout@gmail.com (Corresponding author)

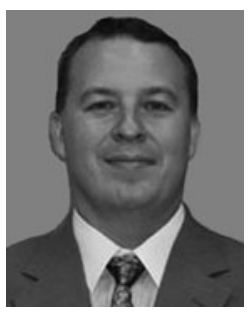

Shannon Bowling received his $\mathrm{Ph} . \mathrm{D}$ degree in industrial engineering with a focus in human factors engineering from Clemson University, USA in 2003. He received his M.Sc. degree in engineering technology with an emphasis in quality management (2000) from East Tennessee State University, USA, and his B.Sc. degree in electrical engineering technology (1998) from Bluefield State College, USA. He also has a graduate minor in experimental statistics from Clemson University and management from East Tennessee State University. He is the Dean of Engineering Technology and Computer Science at Bluefield State College.

He teaches topics relating to modeling and simulation including operations research, optimization methods, systems dynamics, and agent-based modeling. He was a principle investigator (PI) on a project involved in a large scale modeling effort for the Department of Homeland Security. He has various papers published and/or submitted in the area of modeling and simulation. He has over 70 refereed conference and journal publications on various topics.

$\mathrm{He}$ is a member of the Institute of Industrial Engineers (IIE), the Human Factors and Ergonomics Society (HFES), the American Society for Quality (ASQ), and the American Society for Engineering Management (ASEM).

His research interests include the modeling of human performance in complex environments by the use of virtual reality technology, ascertaining factors that influence performance in visual search and decision making tasks, and the analysis of training interventions. He also is interested in using modeling and simulation for decision making in complex environments.

E-mail: srbbsu@gmail.com 